\title{
Radius Constants for Analytic Functions with Fixed Second Coefficient
}

\author{
Mahnaz M. Nargesi, ${ }^{1}$ Rosihan M. Ali, ${ }^{2}$ and V. Ravichandran ${ }^{3}$ \\ ${ }^{1}$ Department of Mathematics, College of Natural Sciences \& Mathematics, California State University, \\ 800 North State College Boulevard, Fullerton, CA 92831-3599, USA \\ ${ }^{2}$ School of Mathematical Sciences, Universiti Sains Malaysia, 11800 Penang, Malaysia \\ ${ }^{3}$ Department of Mathematics, University of Delhi, Delhi 110 007, India
}

Correspondence should be addressed to Rosihan M. Ali; rosihan@cs.usm.my

Received 3 February 2014; Accepted 13 June 2014; Published 1 July 2014

Academic Editor: Ming-Sheng Liu

Copyright (C) 2014 Mahnaz M. Nargesi et al. This is an open access article distributed under the Creative Commons Attribution License, which permits unrestricted use, distribution, and reproduction in any medium, provided the original work is properly cited.

Let $f(z)=z+\sum_{n=2}^{\infty} a_{n} z^{n}$ be analytic in the unit disk with the second coefficient $a_{2}$ satisfying $\left|a_{2}\right|=2 b, 0 \leq b \leq 1$. Sharp radius of Janowski starlikeness is obtained for functions $f$ whose $n$th coefficient satisfies $\left|a_{n}\right| \leq c n+d(c, d \geq 0)$ or $\left|a_{n}\right| \leq c / n$ ( $c>0$ and $n \geq$ 3). Other radius constants are also obtained for these functions, and connections with earlier results are made.

\section{Introduction}

Let $\mathscr{A}$ denote the class of analytic functions $f$ defined in the open unit disk $\mathbb{D}:=\{z \in \mathbb{C}:|z|<1\}$, normalized by $f(0)=$ $0=f^{\prime}(0)-1$, and let $\mathcal{S}$ denote its subclass consisting of univalent functions. If $f(z)=z+\sum_{n=2}^{\infty} a_{n} z^{n} \in \mathcal{S}$, de Branges [1] obtained the sharp coefficient bound that $\left|a_{n}\right| \leq n(n \geq 2)$. However, the inequality $\left|a_{n}\right| \leq n, n \geq 2$, is not sufficient for $f$ to be univalent; for example, $f(z)=z+2 z^{2}$ is clearly not a member of $\mathcal{S}$.

Several subclasses of $\mathcal{S}$ possess a similar coefficient bound. For instance, the $n$th coefficients of starlike functions, convex functions in the direction of imaginary axis, and close-to-convex functions satisfy $\left|a_{n}\right| \leq n(n \geq 2)$ [2-4]. Other examples include functions which are convex, starlike of order $1 / 2$, and starlike with respect to symmetric points. The $n$th coefficients of these functions satisfy $\left|a_{n}\right| \leq 1 \quad(n \geq 2)$ [5-7]. The $n$th coefficient of close-to-convex functions with argument $\beta$ satisfies $\left|a_{n}\right| \leq 1+(n-1) \cos \beta$ [8], and the coefficients of uniformly starlike functions are bounded by $2 / n$ [9], while $\left|a_{n}\right| \leq 1 / n$ [10] for uniformly convex functions. Simple examples show that these bounds are not sufficient to characterize the geometric properties of the classes of functions.
In the sequel, we will assume that $f \in \mathscr{A}$ has the Taylor expansion of the form $f(z)=z+\sum_{n=2}^{\infty} a_{n} z^{n}$. Gavrilov [11] showed that the radius of univalence for functions $f \in \mathscr{A}$ satisfying $\left|a_{n}\right| \leq n(n \geq 2)$ is the real root $r_{0} \simeq 0.164$ of the equation $2(1-r)^{3}-(1+r)=0$, and the result is sharp for $f(z)=2 z-z /(1-z)^{2}$. Gavrilov also proved that the radius of univalence for functions $f \in \mathscr{A}$ satisfying the coefficient bound $\left|a_{n}\right| \leq M(n \geq 2)$ is $1-\sqrt{M /(1+M)}$. The condition $\left|a_{n}\right| \leq M$ clearly holds for functions $f \in \mathscr{A}$ satisfying $|f(z)| \leq$ $M$, and for these functions, Landau [12] proved that the radius of univalence is $M-\sqrt{M^{2}-1}$. In fact, Yamashita [13] showed that the radius of univalence obtained by Gavrilov [11] is also the radius of starlikeness for functions $f \in \mathscr{A}$ satisfying $\left|a_{n}\right| \leq$ $n$ or $\left|a_{n}\right| \leq M$. Additionally, Yamashita [13] determined that the radius of convexity for functions $f \in \mathscr{A}$ satisfying $\left|a_{n}\right| \leq n$ is the real root $r_{0} \simeq 0.090$ of the equation $2(1-r)^{4}-(1+4 r+$ $\left.r^{2}\right)=0$, while the radius of convexity for functions $f \in \mathscr{A}$ satisfying $\left|a_{n}\right| \leq M$ is the real root of

$$
(M+1)(1-r)^{3}-M(1+r)=0 .
$$

Recently, Kalaj et al. [14] obtained the radii of univalence, starlikeness, and convexity for harmonic mappings satisfying certain coefficient inequalities. 
For two analytic functions $f$ and $g$, the function $f$ is subordinate to $g$, denoted by $f \prec g$, if there is an analytic self-map $w$ of $\mathbb{D}$ with $w(0)=0$ satisfying $f(z)=g(w(z))$. If $g$ is univalent, then $f \prec g$ is equivalent to $f(0)=g(0)$ and $f(\mathbb{D}) \subseteq g(\mathbb{D})$.

For $\beta \in \mathbb{R} \backslash\{1\}, \alpha \geq 0$, the class $\mathscr{L}(\alpha, \beta)$ consists of functions $f \in \mathscr{A}$ satisfying

$$
\alpha \frac{z^{2} f^{\prime \prime}(z)}{f(z)}+\frac{z f^{\prime}(z)}{f(z)} \prec \frac{1+(1-2 \beta) z}{1-z} .
$$

Denote by $\mathscr{L}_{0}(\alpha, \beta)$ its subclass consisting of functions $f \in \mathscr{A}$ satisfying

$$
\left|\alpha \frac{z^{2} f^{\prime \prime}(z)}{f(z)}+\frac{z f^{\prime}(z)}{f(z)}-1\right| \leq|1-\beta| \quad(\beta \in \mathbb{R} \backslash\{1\}, \alpha \geq 0) .
$$

These classes were investigated in [15-24].

For $\beta<1$, the class $\mathscr{L}(0, \beta)$ is the class of starlike functions of order $\beta$, while, for the case $\beta>1$, the class was studied in [25-28].

The class $\mathcal{S} \mathscr{T}[A, B]$ of Janowski starlike functions [29] consists of $f \in \mathscr{A}$ satisfying the subordination

$$
\frac{z f^{\prime}(z)}{f(z)} \prec \frac{1+A z}{1+B z} \quad(-1 \leq B<A \leq 1) .
$$

Certain well-known subclasses of starlike functions are special cases of $\mathcal{S} \mathscr{T}[A, B]$ for appropriate choices of the parameters $A$ and $B$. For example, for $0 \leq \beta<1, \mathcal{S} \mathscr{T}(\beta):=$ $\mathcal{S} \mathscr{T}[1-2 \beta,-1]$ is the familiar class of starlike functions of order $\beta$. Denote by $\mathcal{S} \mathscr{T}_{\beta}$ the class $\mathcal{S} \mathscr{T}_{\beta}:=\mathscr{L}_{0}(0, \beta)=$ $\mathcal{S} \mathscr{T}[1-\beta, 0]$. Janowski [29] obtained the sharp radius of convexity for $\mathcal{S} \mathscr{T}[A, B]$.

This paper studies the class $\mathscr{A}_{b}$ consisting of functions $f(z)=z+\sum_{n=2}^{\infty} a_{n} z^{n}, \quad\left(\left|a_{2}\right|=2 b, 0 \leq b \leq 1\right)$, in the disk $\mathbb{D}$. The subclass of univalent functions in $\mathscr{A}_{b}$ have been studied in [30-33]. In [33], Ravichandran obtained sharp radii of starlikeness and convexity of order $\alpha$ for functions $f \in \mathscr{A}_{b}$ satisfying $\left|a_{n}\right| \leq n$ or $\left|a_{n}\right| \leq M, n \geq 3$. The author also obtained the radius of uniform convexity and parabolic starlikeness for functions $f \in \mathscr{A}_{b}$ satisfying $\left|a_{n}\right| \leq n, n \geq 3$.

This paper finds radius constants for functions $f(z)=$ $z+\sum_{n=2}^{\infty} a_{n} z^{n} \in \mathscr{A}_{b}$ satisfying either $\left|a_{n}\right| \leq c n+d(c, d \geq 0)$ or $\left|a_{n}\right| \leq c / n(c>0, n \geq 3)$. In the next section, sharp $\mathscr{L}(\alpha, \beta)$ radius and $\mathcal{S} \mathscr{T}[A, B]$-radius are derived for these classes. Several known radius constants are shown to be special cases of the results obtained.

\section{Radius Constants}

A sufficient condition for functions $f \in \mathscr{A}$ to belong to the class $\mathscr{L}(\alpha, \beta)$ is given in the following lemma.

Lemma 1 (see $[24,34]$ ). Let $\beta \in \mathbb{R} \backslash\{1\}$ and $\alpha \geq 0$. If $f(z)=$ $z+\sum_{n=2}^{\infty} a_{n} z^{n} \in \mathscr{A}$ satisfies the inequality

$$
\sum_{n=2}^{\infty}\left(\alpha n^{2}+(1-\alpha) n-\beta\right)\left|a_{n}\right| \leq|1-\beta|,
$$

then $f \in \mathscr{L}(\alpha, \beta)$.
Making use of this lemma, the sharp $\mathscr{L}(\alpha, \beta)$-radius is obtained for $f \in \mathscr{A}_{b}$ satisfying the coefficient inequality $\left|a_{n}\right| \leq c n+d$.

Theorem 2. Let $\beta \in \mathbb{R} \backslash\{1\}, 6 \alpha+3-\beta \geq 0$, and $\alpha \geq 0$. The $\mathscr{L}(\alpha, \beta)$-radius for $f(z)=z+\sum_{n=2}^{\infty} a_{n} z^{n} \in \mathscr{A}_{b}$ satisfying the coefficient inequality $\left|a_{n}\right| \leq c n+d, c, d \geq 0, n \geq 3$, is the real root in $(0,1)$ of the equation

$$
\begin{aligned}
& ((c+d)(1-\beta)+|1-\beta| \\
& +(2 \alpha+2-\beta)(2(c-b)+d) r)(1-r)^{4} \\
& =c \alpha\left(1+4 r+r^{2}\right)+((1-\alpha) c+\alpha d)\left(1-r^{2}\right) \\
& +((1-\alpha) d-\beta c)(1-r)^{2}-\beta d(1-r)^{3} .
\end{aligned}
$$

For $\beta<1$, this number is also the $\mathscr{L}_{0}(\alpha, \beta)$-radius of $f \in \mathscr{A}_{b}$. The results are sharp.

Proof. The number $r_{0}$ is the $\mathscr{L}(\alpha, \beta)$-radius for $f \in \mathscr{A}_{b}$ if and only if $f\left(r_{0} z\right) / r_{0} \in \mathscr{L}(\alpha, \beta)$. Therefore, by Lemma 1 , it is sufficient to verify the inequality

$$
\sum_{n=2}^{\infty}\left(\alpha n^{2}+(1-\alpha) n-\beta\right)\left|a_{n}\right| r_{0}^{n-1} \leq|1-\beta|,
$$

where $r_{0}$ is the real root in $(0,1)$ of $(6)$. Using the known expansions

$$
\begin{aligned}
\sum_{n=3}^{\infty} r_{0}^{n-1} & =\frac{1}{1-r_{0}}-1-r_{0}, \\
\sum_{n=3}^{\infty} n r_{0}^{n-1} & =\frac{1}{\left(1-r_{0}\right)^{2}}-1-2 r_{0}, \\
\sum_{n=3}^{\infty} n^{2} r_{0}^{n-1} & =\frac{1+r_{0}}{\left(1-r_{0}\right)^{3}}-1-4 r_{0}, \\
\sum_{n=3}^{\infty} n^{3} r_{0}^{n-1} & =\frac{1+4 r_{0}+r_{0}^{2}}{\left(1-r_{0}\right)^{4}}-1-8 r_{0}
\end{aligned}
$$

leads to

$$
\begin{aligned}
\sum_{n=2}^{\infty}( & \left.\alpha n^{2}+(1-\alpha) n-\beta\right)\left|a_{n}\right| r_{0}^{n-1} \\
\leq & 2(2 \alpha+2-\beta) b r_{0} \\
& +\sum_{n=3}^{\infty}\left(\alpha n^{2}+(1-\alpha) n-\beta\right)(c n+d) r_{0}^{n-1} \\
= & 2(2 \alpha+2-\beta) b r_{0}+c \alpha\left(\frac{1+4 r_{0}+r_{0}^{2}}{\left(1-r_{0}\right)^{4}}-1-8 r_{0}\right) \\
& +((1-\alpha) c+\alpha d)\left(\frac{1+r_{0}}{\left(1-r_{0}\right)^{3}}-1-4 r_{0}\right) \\
& +((1-\alpha) d-\beta c)\left(\frac{1}{\left(1-r_{0}\right)^{2}}-1-2 r_{0}\right) \\
& -\beta d\left(\frac{1}{1-r_{0}}-1-r_{0}\right)
\end{aligned}
$$




$$
\begin{aligned}
& =(c+d)(\beta-1)-(2 \alpha+2-\beta)(2(c-b)+d) r_{0} \\
& +\left(c \alpha\left(1+4 r_{0}+r_{0}^{2}\right)+((1-\alpha) c+\alpha d)\left(1-r_{0}^{2}\right)\right. \\
& \quad+((1-\alpha) d-\beta c)\left(1-r_{0}\right)^{2} \\
& \left.\quad-\beta d\left(1-r_{0}\right)^{3}\right) \times\left(1-r_{0}\right)^{-4} \\
& \quad|1-\beta| .
\end{aligned}
$$

For $\beta<1$, consider the function

$$
\begin{aligned}
f_{0}(z) & =z-2 b z^{2}-\sum_{n=3}^{\infty}(c n+d) z^{n} \\
& =(c+1) z+2(c-b) z^{2}-\frac{c z}{(1-z)^{2}}-\frac{d z^{3}}{1-z} .
\end{aligned}
$$

At the root $z=r_{0}$ in $(0,1)$ of $(6), f_{0}$ satisfies

$$
\operatorname{Re}\left(\alpha \frac{z^{2} f_{0}^{\prime \prime}(z)}{f_{0}(z)}+\frac{z f_{0}^{\prime}(z)}{f_{0}(z)}\right)=1-\frac{N\left(r_{0}\right)}{D\left(r_{0}\right)}=\beta,
$$

where

$$
\begin{aligned}
N\left(r_{0}\right)= & -2(c-b)(2 \alpha+1) r_{0}+\frac{2 c r_{0}(2 \alpha+1)}{\left(1-r_{0}\right)^{3}} \\
& +\frac{6 c \alpha r_{0}^{2}}{\left(1-r_{0}\right)^{4}}+\frac{2 d r_{0}^{2}(3 \alpha+1)}{1-r_{0}} \\
& +\frac{d r_{0}^{3}(6 \alpha+1)}{\left(1-r_{0}\right)^{2}}+\frac{2 d r_{0}^{4} \alpha}{\left(1-r_{0}\right)^{3}}, \\
D\left(r_{0}\right)=c & +1+2(c-b) r_{0}-\frac{c}{\left(1-r_{0}\right)^{2}}-\frac{d r_{0}^{2}}{1-r_{0}}
\end{aligned}
$$

This shows that $r_{0}$ is the sharp $\mathscr{L}(\alpha, \beta)$-radius for $f \in \mathscr{A}_{b}$. For $\beta<1$, (14) shows that the rational expression $N\left(r_{0}\right) / D\left(r_{0}\right)$ is positive, and therefore the equality

$$
\left|\alpha \frac{z^{2} f_{0}^{\prime \prime}(z)}{f_{0}(z)}+\frac{z f_{0}^{\prime}(z)}{f_{0}(z)}-1\right|=1-\beta
$$

holds. Thus, $r_{0}$ is the sharp $\mathscr{L}_{0}(\alpha, \beta)$-radius for $f \in \mathscr{A}_{b}$ when $\beta<1$.

For $\beta>1$, the function

$$
\begin{aligned}
f_{0}(z) & =z+2 b z^{2}+\sum_{n=3}^{\infty}(c n+d) z^{n} \\
& =(1-c) z+2(b-c) z^{2}+\frac{c z}{(1-z)^{2}}+\frac{d z^{3}}{1-z}
\end{aligned}
$$

demonstrates sharpness of the result. The derivation is similar to the case $\beta<1$ and is omitted.
Theorem 3. Let $\beta \in \mathbb{R} \backslash\{1\}$ and $\alpha \geq 0$. The $\mathscr{L}(\alpha, \beta)$-radius of $f(z)=z+\sum_{n=2}^{\infty} a_{n} z^{n} \in \mathscr{A}_{b}$ satisfying the coefficient inequality $\left|a_{n}\right| \leq c / n$ for $n \geq 3$ and $c>0$ is the real root in $(0,1)$ of the equation

$$
\begin{array}{r}
{\left[c(1-\beta)+|1-\beta|+(2 \alpha+2-\beta) r\left(\frac{c}{2}-2 b\right)\right](1-r)^{2}} \\
=c \alpha+(1-\alpha) c(1-r)+\beta c(1-r)^{2} \frac{\log (1-r)}{r} .
\end{array}
$$

For $\beta<1$, this number is also the $\mathscr{L}_{0}(\alpha, \beta)$-radius of $f \in \mathscr{A}_{b}$. The results are sharp.

Proof. By Lemma $1, r_{0}$ is the $\mathscr{L}(\alpha, \beta)$-radius of functions $f \in$ $\mathscr{A}_{b}$ when inequality (7) holds for the real root $r_{0}$ of (18) in $(0,1)$. Using (8) and (9) together with

$$
\sum_{n=3}^{\infty} \frac{r_{0}^{n-1}}{n}=-\frac{\log \left(1-r_{0}\right)}{r_{0}}-1-\frac{r_{0}}{2}
$$

leads to

$$
\begin{aligned}
\sum_{n=2}^{\infty}( & \left.\alpha n^{2}+(1-\alpha) n-\beta\right)\left|a_{n}\right| r_{0}^{n-1} \\
\leq & 2(2 \alpha+2-\beta) b r_{0} \\
& +\sum_{n=3}^{\infty}\left(\alpha n^{2}+(1-\alpha) n-\beta\right)\left(\frac{c}{n}\right) r_{0}^{n-1} \\
= & 2(2 \alpha+2-\beta) b r_{0}+c \alpha\left(\frac{1}{\left(1-r_{0}\right)^{2}}-1-2 r_{0}\right) \\
& +(1-\alpha) c\left(\frac{1}{1-r_{0}}-1-r_{0}\right) \\
& -\beta c\left(-\frac{\log \left(1-r_{0}\right)}{r_{0}}-1-\frac{r_{0}}{2}\right) \\
= & c(\beta-1)+(2 \alpha+2-\beta) r_{0}\left(2 b-\frac{c}{2}\right) \\
= & |1-\beta| . \\
& +\frac{c \alpha r_{0}+(1-\alpha) c\left(1-r_{0}\right) r_{0}+\beta c\left(1-r_{0}\right)^{2} \log \left(1-r_{0}\right)}{\left(1-r_{0}\right)^{2} r_{0}} \\
&
\end{aligned}
$$

To verify sharpness for $\beta<1$, consider the function

$$
\begin{aligned}
f_{0}(z) & =z-2 b z^{2}-\sum_{n=3}^{\infty} \frac{c}{n} z^{n} \\
& =(1+c) z+\left(\frac{c}{2}-2 b\right) z^{2}+c \log (1-z) .
\end{aligned}
$$


At the root $z=r_{0}$ in $(0,1)$ of $(18), f_{0}$ satisfies

$$
\begin{aligned}
& \operatorname{Re}\left(\alpha \frac{z^{2} f_{0}^{\prime \prime}(z)}{f_{0}(z)}+\frac{z f_{0}^{\prime}(z)}{f_{0}(z)}\right) \\
& =1-\left(-\left(\frac{c}{2}-2 b\right) r_{0}(2 \alpha+1)+\frac{c r_{0} \alpha}{\left(1-r_{0}\right)^{2}}\right. \\
& \left.\quad+\frac{c}{1-r_{0}}+\frac{c \log \left(1-r_{0}\right)}{r_{0}}\right) \\
& \quad \times\left((1+c)+\left(\frac{c}{2}-2 b\right) r_{0}+\frac{c \log \left(1-r_{0}\right)}{r_{0}}\right)^{-1}=\beta
\end{aligned}
$$

Thus, $r_{0}$ is the sharp $\mathscr{L}(\alpha, \beta)$-radius for $f \in \mathscr{A}_{b}$. For $\beta<1$, the rational expression in (22) is positive, and therefore

$$
\left|\alpha \frac{z^{2} f_{0}^{\prime \prime}(z)}{f_{0}(z)}+\frac{z f_{0}^{\prime}(z)}{f_{0}(z)}-1\right|=1-\beta,
$$

which shows that $r_{0}$ is the sharp $\mathscr{L}_{0}(\alpha, \beta)$-radius for $f \in \mathscr{A}_{b}$. For $\beta>1$, sharpness of the result is demonstrated by the function $f_{0}$ given by

$$
\begin{aligned}
f_{0}(z) & =z+2 b z^{2}+\sum_{n=3}^{\infty} \frac{c}{n} z^{n} \\
& =(1-c) z+\left(2 b-\frac{c}{2}\right) z^{2}-c \log (1-z) .
\end{aligned}
$$

Remark 4. The results obtained above yield the following special cases.

(1) For $\alpha=0, \beta=0, c=1, d=0$, and $0 \leq b \leq 1$, Theorem 2 yields the radius of starlikeness obtained by Yamashita [13].

(2) For $\alpha=0, c=1$, and $d=0$, Theorem 2 reduces to Theorem 2.1 in [33, page 3]. When $\alpha=0, c=0$, and $d=M$, Theorem 2 leads to Theorem 2.5 in [33, page 5].

(3) For $\alpha=0$, Theorem 3 yields the radius of starlikeness of order $\beta$ for $f \in \mathscr{A}_{b}$ obtained by Ravichandran [33, Theorem 2.8].

The following result of Goel and Sohi [35] will be required in our investigation of the class of Janowski starlike functions.

Lemma 5 (see [35]). Let $-1 \leq B<A \leq 1$. If $f(z)=z+$ $\sum_{n=2}^{\infty} a_{n} z^{n} \in \mathscr{A}$ satisfies the inequality

$$
\sum_{n=2}^{\infty}((1-B) n-(1-A))\left|a_{n}\right| \leq A-B,
$$

then $f \in \mathcal{S} \mathscr{T}[A, B]$.

The next result finds the sharp $\mathcal{S} \mathscr{T}[A, B]$-radius for $f \in$ $\mathscr{A}_{b}$ satisfying the coefficient inequality $\left|a_{n}\right| \leq c n+d$.
Theorem 6. Let $-1 \leq B<A \leq 1$. The $\mathcal{S} \mathscr{T}[A, B]$-radius for $f(z)=z+\sum_{n=2}^{\infty} a_{n} z^{n} \in \mathscr{A}_{b}$ satisfying the coefficient inequality $\left|a_{n}\right| \leq c n+d, n \geq 3$ and $c, d \geq 0$, is the real root in $(0,1)$ of the equation

$$
\begin{aligned}
& {[(A-B)(c+d+1)} \\
& -(2 b-2 c-d)(2(1-B)-(1-A)) r](1-r)^{3} \\
& =c(1-B)(1+r)+(d(1-B)-c(1-A))(1-r) \\
& \quad-(1-A) d(1-r)^{2} .
\end{aligned}
$$

This radius is sharp.

Proof. It is evident that $r_{0}$ is the $\mathcal{S} \mathscr{T}[A, B]$-radius of $f \in \mathscr{A}_{b}$ if and only if $f\left(r_{0} z\right) / r_{0} \in \mathcal{S} \mathscr{T}[A, B]$. Hence, by Lemma 5 , it suffices to show that

$$
\begin{array}{r}
\sum_{n=2}^{\infty}((1-B) n-(1-A))\left|a_{n}\right| r_{0}^{n-1} \leq A-B \\
(-1 \leq B<A \leq 1),
\end{array}
$$

where $r_{0}$ is the root in $(0,1)$ of $(26)$. From (8), (9), and (10), it follows that

$$
\begin{aligned}
& \sum_{n=2}^{\infty}((1-B) n-(1-A))\left|a_{n}\right| r_{0}^{n-1} \\
& \leq 2(2(1-B)-(1-A)) b r_{0} \\
& +\sum_{n=3}^{\infty}((1-B) n-(1-A))(c n+d) r_{0}^{n-1} \\
& =2(2(1-B)-(1-A)) b r_{0} \\
& +c(1-B)\left(\frac{1+r_{0}}{\left(1-r_{0}\right)^{3}}-1-4 r_{0}\right) \\
& +(d(1-B)-c(1-A))\left(\frac{1}{\left(1-r_{0}\right)^{2}}-1-2 r_{0}\right) \\
& -(1-A) d\left(\frac{1}{1-r_{0}}-1-r_{0}\right) \\
& =(B-A)(c+d)+(2 b-2 c-d) \\
& \times(2(1-B)-(1-A)) r_{0} \\
& +\left(c(1-B)\left(1+r_{0}\right)\right. \\
& +(d(1-B)-c(1-A))\left(1-r_{0}\right) \\
& \left.-(1-A) d\left(1-r_{0}\right)^{2}\right) \times\left(1-r_{0}\right)^{-3} \\
& =A-B \text {. }
\end{aligned}
$$


The function $f_{0}$ given by (13) shows that the result is sharp. Indeed, at the point $z=r_{0}$ where $r_{0}$ is the root in $(0,1)$ of (26), the function $f_{0}$ satisfies

$$
\begin{aligned}
\left|\frac{z f_{0}^{\prime}(z)}{f_{0}(z)}-1\right| & \left(-2(c-b) r_{0}+\frac{2 d r_{0}^{2}}{1-r_{0}}+\frac{d r_{0}^{3}}{\left(1-r_{0}\right)^{2}}+\frac{2 c r_{0}}{\left(1-r_{0}\right)^{3}}\right) \\
= & \times\left(c+1+2(c-b) r_{0}-\frac{c}{\left(1-r_{0}\right)^{2}}-\frac{d r_{0}^{2}}{1-r_{0}}\right)^{-1}, \\
A-B \frac{z f_{0}^{\prime}(z)}{f_{0}(z)} \mid & \frac{(c+1)(A-B)+2(c-b) r_{0}(A-2 B)}{c+1+2(c-b) r_{0}-c /\left(1-r_{0}\right)^{2}-d r_{0}^{2} /\left(1-r_{0}\right)} \\
& -\left(\frac{c(A-B)}{\left(1-r_{0}\right)^{2}}+\frac{2 c r_{0} B}{\left(1-r_{0}\right)^{3}}\right. \\
& \times\left(\begin{array}{c}
\left.c+1+2(c-b) r_{0}-\frac{c}{\left(1-r_{0}\right)^{2}}-\frac{d r_{0}^{2}}{1-r_{0}}\right)^{-1} \\
\left.1-r_{0}+\frac{d r_{0}^{3} B}{\left(1-r_{0}\right)^{2}}\right)
\end{array}\right.
\end{aligned}
$$

Then, (26) yields

$$
\left|\frac{z f_{0}^{\prime}(z)}{f_{0}(z)}-1\right|=\left|A-B \frac{z f_{0}^{\prime}(z)}{f_{0}(z)}\right| \quad\left(-1 \leq B<A \leq 1, z=r_{0}\right),
$$

or equivalently $f_{0} \in \mathcal{S} \mathscr{T}[A, B]$.

Theorem 7. Let $-1 \leq B<A \leq 1$. The $\mathcal{S} \mathscr{T}[A, B]$-radius for $f(z)=z+\sum_{n=2}^{\infty} a_{n} z^{n} \in \mathscr{A}_{b}$ satisfying the coefficient inequality $\left|a_{n}\right| \leq c / n, n \geq 3$ and $c>0$, is the real root in $(0,1)$ of the equation

$$
\begin{aligned}
& \left((c+1)(A-B)-(2(1-B)-(1-A)) r\left(2 b-\frac{c}{2}\right)\right) \\
& \quad \times(1-r) \\
& =c(1-B)+c(1-A)(1-r) \frac{\log (1-r)}{r} .
\end{aligned}
$$

This radius is sharp.
Proof. By Lemma 5, condition (27) assures that $r_{0}$ is the $\mathcal{S T}[A, B]$-radius of $f \in \mathscr{A}_{b}$ where $r_{0}$ is the real root of (31). Therefore, using (8) and (19) for $f \in \mathscr{A}_{b}$ yields

$$
\begin{aligned}
\sum_{n=2}^{\infty}( & (1-B) n-(1-A))\left|a_{n}\right| r_{0}^{n-1} \\
\leq & 2(2(1-B)-(1-A)) b r_{0} \\
& +\sum_{n=3}^{\infty}((1-B) n-(1-A))\left(\frac{c}{n}\right) r_{0}^{n-1} \\
= & 2(2(1-B)-(1-A)) b r_{0} \\
& +c(1-B)\left(\frac{1}{1-r_{0}}-1-r_{0}\right) \\
& -c(1-A)\left(-\frac{\log \left(1-r_{0}\right)}{r_{0}}-1-\frac{r_{0}}{2}\right) \\
= & c(B-A)+(2(1-B)-(1-A)) r_{0}\left(2 b-\frac{c}{2}\right) \\
& +\frac{c(1-B) r_{0}+c(1-A)\left(1-r_{0}\right) \log \left(1-r_{0}\right)}{\left(1-r_{0}\right) r_{0}} \\
= & A-B .
\end{aligned}
$$

The result is sharp for the function $f_{0}$ given by (21). Indeed, $f_{0}$ satisfies

$$
\begin{aligned}
& \left|\frac{z f_{0}^{\prime}(z)}{f_{0}(z)}-1\right| \\
& =\frac{-(c / 2-2 b) r_{0}+c /\left(1-r_{0}\right)+\left(c \log \left(1-r_{0}\right)\right) / r_{0}}{(1+c)+(c / 2-2 b) r_{0}+\left(c \log \left(1-r_{0}\right)\right) / r_{0}}, \\
& \left|A-B \frac{z f_{0}^{\prime}(z)}{f_{0}(z)}\right| \\
& =\left((1+c)(A-B)+(A-2 B)\left(\frac{c}{2}-2 b\right) r_{0}\right. \\
& \left.\quad+\frac{c B}{1-r_{0}}+\frac{c A \log \left(1-r_{0}\right)}{r_{0}}\right) \\
& \quad \times\left((1+c)+\left(\frac{c}{2}-2 b\right) r_{0}+\frac{c \log \left(1-r_{0}\right)}{r_{0}}\right)^{-1},
\end{aligned}
$$

at the root $z=r_{0}$ in $(0,1)$ of $(31)$. Evidently, the function $f_{0}$ satisfies (30), and hence the result is sharp.

\section{Conflict of Interests}

The authors declare that there is no conflict of interests regarding the publication of this paper. 


\section{Acknowledgments}

The work presented here was supported in parts by an FRGS Grant 203/PMATHS/6711366 and a grant from the University of Delhi.

\section{References}

[1] L. de Branges, "A proof of the Bieberbach conjecture," Acta Mathematica, vol. 154, no. 1-2, pp. 137-152, 1985.

[2] R. Nevanlinna, "Uber die konforme Abbildung Sterngebieten," Oeversikt av Finska-Vetenskaps Societeten Forhandlingar A, vol. 63, no. 6, pp. 1-21, 1921.

[3] A. W. Goodman, Univalent Functions, vol. 1, Mariner Publishing, Tampa, Fla, USA, 1983.

[4] M. O. Reade, "On close-to-close univalent functions," The Michigan Mathematical Journal, vol. 3, pp. 59-62, 1955.

[5] C. Lowener, "Untersuchungen uber die Verzerrung bei konformen Abbildungen des Einheitskreises $|z|<1$," Leipzing Berichte, vol. 69, pp. 89-106, 1917.

[6] A. Schild, "On a class of univalent, star shaped mappings," Proceedings of the American Mathematical Society, vol. 9, pp. 751-757, 1958.

[7] K. Sakaguchi, "On a certain univalent mapping," Journal of the Mathematical Society of Japan, vol. 11, pp. 72-75, 1959.

[8] A. W. Goodman and E. B. Saff, "On the definition of a closeto-convex function," International Journal of Mathematics and Mathematical Sciences, vol. 1, no. 1, pp. 125-132, 1978.

[9] A. W. Goodman, “On uniformly starlike functions," Journal of Mathematical Analysis and Applications, vol. 155, no. 2, pp. 364370, 1991.

[10] A. W. Goodman, "On uniformly convex functions," Annales Polonici Mathematici, vol. 56, no. 1, pp. 87-92, 1991.

[11] V. I. Gavrilov, "Remarks on the radius of univalence of holomorphic functions," Matematicheskie Zametki, vol. 7, pp. 295-298, 1970.

[12] E. Landau, "Der Picard-Schottkysche Satz und die Blochsche Konstante," Sitzungsberichte der Preussischen Akademie der Wissenschaften. Physikalisch-Mathematische Klasse, pp. 467474, 1925.

[13] S. Yamashita, "Radii of univalence, starlikeness, and convexity," Bulletin of the Australian Mathematical Society, vol. 25, no. 3, pp. 453-457, 1982.

[14] D. Kalaj, S. Ponnusamy, and M. Vuorinen, "Radius of closeto-convexity and fully starlikeness of harmonic mappings," Complex Variables and Elliptic Equations, vol. 59, no. 4, pp. 539552, 2014.

[15] Z. W. Liu and M. S. Liu, "Properties and characteristics of certain subclass of analytic functions," Journal of South China Normal University: Natural Science Edition, no. 3, pp. 11-14, 18, 2010.

[16] Z. Lewandowski, S. Miller, and E. Zlotkiewicz, "Generating functions for some classes of univalent functions," Proceedings of the American Mathematical Society, vol. 56, pp. 111-117, 1976.

[17] C. Ramesha, S. Kumar, and K. S. Padmanabhan, "A sufficient condition for starlikeness," Chinese Journal of Mathematics, vol. 23, no. 2, pp. 167-171, 1995.

[18] M. Nunokawa, S. Owa, S. K. Lee et al., "Sufficient conditions for starlikeness," Chinese Journal of Mathematics, vol. 24, no. 3, pp. 265-271, 1996.
[19] M. Obradovic and S. B. Joshi, "On certain classes of strongly starlike functions," Taiwanese Journal of Mathematics, vol. 2, no. 3, pp. 297-302, 1998.

[20] J. Li and S. Owa, "Sufficient conditions for starlikeness," Indian Journal of Pure and Applied Mathematics, vol. 33, no. 3, pp. 313318, 2002.

[21] K. S. Padmanabhan, "On sufficient conditions for starlikeness," Indian Journal of Pure and Applied Mathematics, vol. 32, no. 4, pp. 543-550, 2001.

[22] V. Ravichandran, C. Selvaraj, and R. Rajalaksmi, "Sufficient conditions for starlike functions of order $\alpha$," Journal of Inequalities in Pure and Applied Mathematics, vol. 3, no. 5, article 81, 6 pages, 2002.

[23] V. Ravichandran, "Certain applications of first order differential subordination," Far East Journal of Mathematical Sciences (FJMS), vol. 12, no. 1, pp. 41-51, 2004.

[24] M. S. Liu, Y. C. Zhu, and H. M. Srivastava, "Properties and characteristics of certain subclasses of starlike functions of order $\beta$," Mathematical and Computer Modelling, vol. 48, no. 34, pp. 402-419, 2008.

[25] J. Nishiwaki and S. Owa, "Coefficient inequalities for certain analytic functions," International Journal of Mathematics and Mathematical Sciences, vol. 29, no. 5, pp. 285-290, 2002.

[26] B. A. Uralegaddi, M. D. Ganigi, and S. M. Sarangi, "Univalent functions with positive coefficients," Tamkang Journal of Mathematics, vol. 25, no. 3, pp. 225-230, 1994.

[27] B. A. Uralegaddi and A. R. Desai, "Convolutions of univalent functions with positive coefficients," Tamkang Journal of Mathematics, vol. 29, no. 4, pp. 279-285, 1998.

[28] S. Owa and H. M. Srivastava, "Some generalized convolution properties associated with certain subclasses of analytic functions," Journal of Inequalities in Pure and Applied Mathematics, vol. 3, no. 3, article 42, 13 pages, 2002.

[29] W. Janowski, "Some extremal problems for certain families of analytic functions-I," Annales Polonici Mathematici, vol. 28, pp. 297-326, 1973.

[30] R. M. Ali, N. E. Cho, and N. K. a. Jain, "Radii of starlikeness and convexity for functions with fixed second coefficient defined by subordination," Filomat, vol. 26, no. 3, pp. 553-561, 2012.

[31] R. M. Ali, S. Nagpal, and V. Ravichandran, "Second-order differential subordination for analytic functions with fixed initial coefficient," Bulletin of the Malaysian Mathematical Sciences Society, vol. 34, no. 3, pp. 611-629, 2011.

[32] S. Nagpal and V. Ravichandran, "Applications of the theory of differential subordination for functions with fixed initial coefficient to univalent functions," Annales Polonici Mathematici, vol. 105, no. 3, pp. 225-238, 2012.

[33] V. Ravichandran, "Radii of starlikeness and convexity of analytic functions satisfying certain coefficient inequalities," Mathematica Slovaca, vol. 64, no. 1, pp. 27-38, 2014.

[34] Y. Sun, Z. Wang, and R. Xiao, "Neighbourhoods and partial sums of certain subclass of analytic functions," Acta Universitatis Apulensis. Mathematics-Informatics, no. 26, pp. 217-224, 2011.

[35] R. M. Goel and N. S. Sohi, "Multivalent functions with negative coefficients," Indian Journal of Pure and Applied Mathematics, vol. 12, no. 7, pp. 844-853, 1981. 


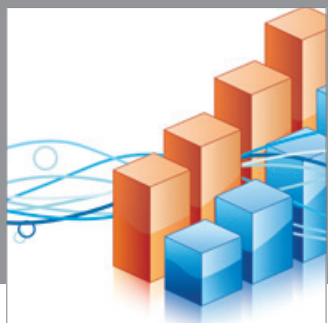

Advances in

Operations Research

mansans

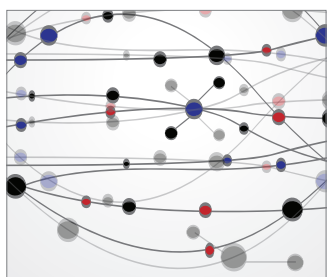

The Scientific World Journal
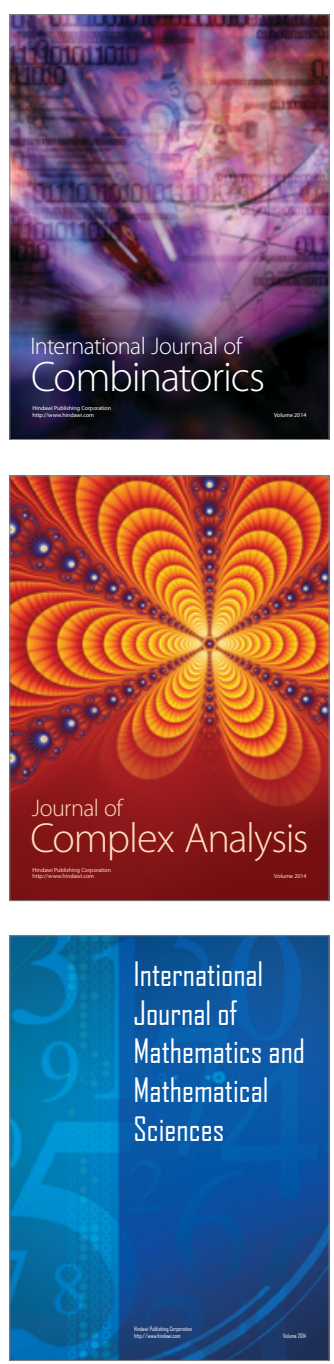
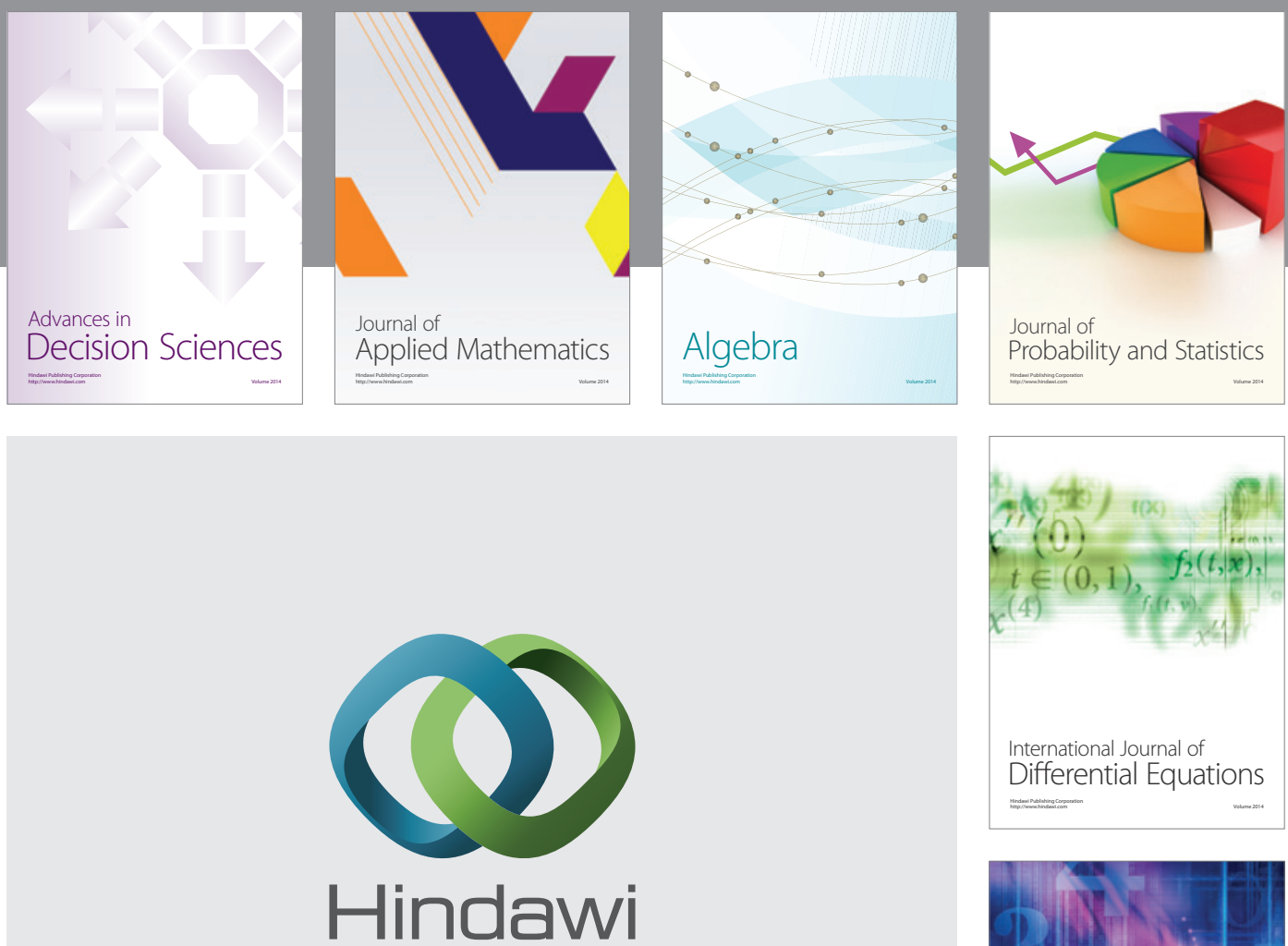

Submit your manuscripts at http://www.hindawi.com
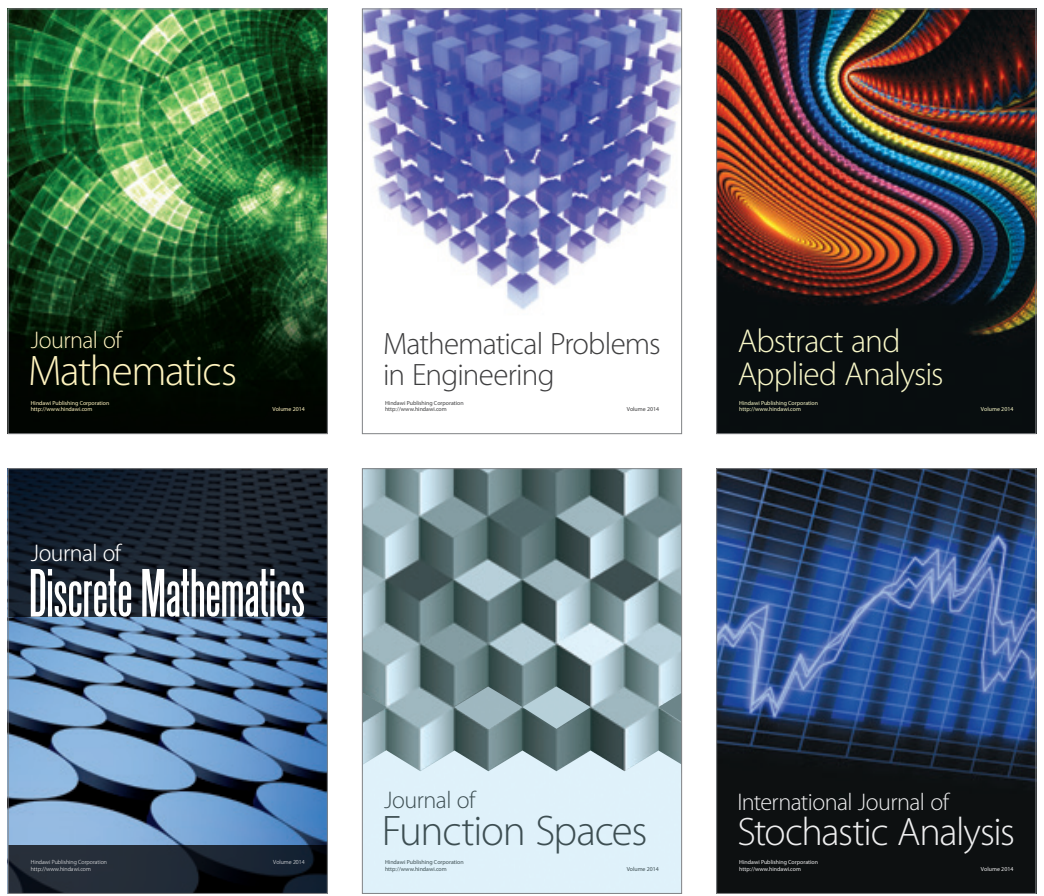

Journal of

Function Spaces

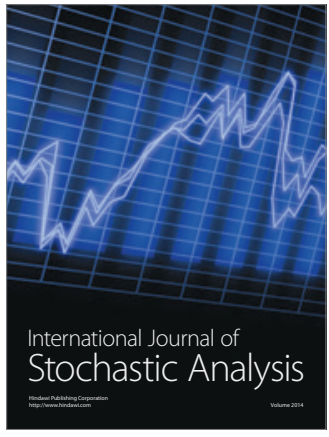

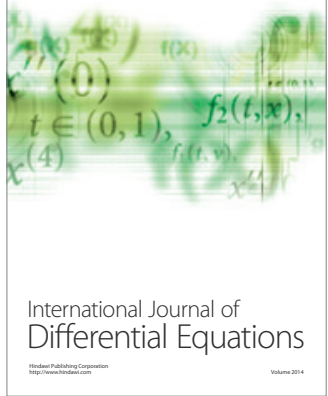
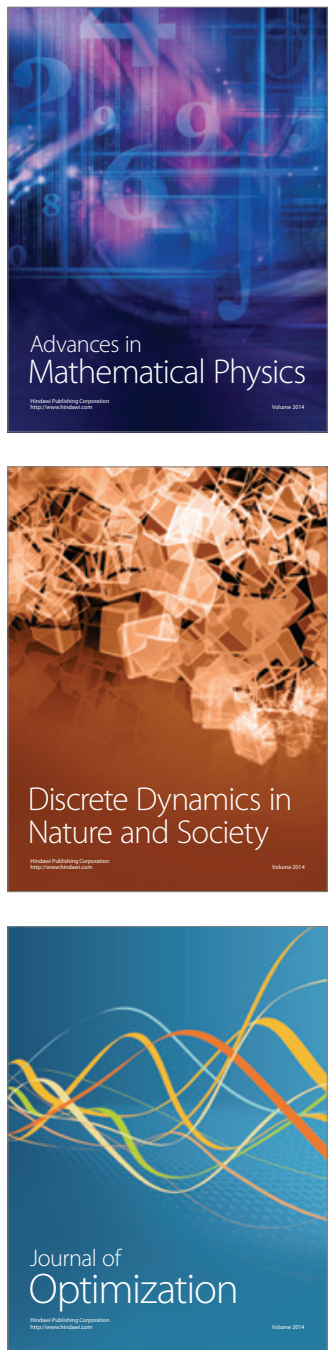